\title{
Prevalência da fragilidade em idosos institucionalizados no município de Rio Verde -
}

\section{Goiás}

\author{
Prevalence of frailty in institutionalized elderly in the city of Rio Verde - Goiás \\ Prevalencia de fragilidad en ancianos institucionalizados de la ciudad de Río Verde - Goiás
}

Recebido: 09/01/2022 | Revisado: 14/01/2022 | Aceito: 15/01/2022 | Publicado: 18/01/2022

\author{
Ana Paula Felix Arantes \\ ORCID: https://orcid.org/0000-0002-7147-7346 \\ Universidade de Rio Verde, Brasil \\ E-mail: anapaulaarantes@unirv.edu.br \\ Valquíria Miranda Silva \\ ORCID: https://orcid.org/0000-0002-5850-8195 \\ Hospital América, Brasil \\ E-mail: valquiriams94@gmail.com \\ Vanessa da Silva Carvalho Vila \\ ORCID: https://orcid.org/0000-0002-1785-8682 \\ Pontifícia Universidade Católica de Goiás, Brasil \\ E-mail:vscvila@uol.com.br \\ Priscila Valverde de Oliveira Vitorino \\ ORCID: https://orcid.org/0000-0002-5487-4649 \\ Pontifícia Universidade Católica de Goiás, Brasil \\ E-mail: pvalverde@ @ucgoias.edu.br
}

\begin{abstract}
Resumo
Objetivo: determinar a prevalência de fragilidade em idosos residentes em uma instituição de longa permanência. Método: estudo transversal descritivo realizado com idosos residentes em uma instituição de longa permanência. Foram utilizados questionários para avaliação das características sociodemográficas e econômicas e Escala de Fragilidade de Edmonton. Resultados: Entre os 75 idosos institucionalizados, 81,33\% foram considerados frágeis. Constatou-se que a faixa etária de 60 a 79 anos e aposentados apresentaram relação significativamente relevante com a fragilidade. Conclusão: a maioria dos idosos avaliados apresentou fragilidade, a qual é uma síndrome de caráter multidimensional, impacta na vida pessoal, familiar e social dos idosos, pode ser uma condição precursora do declínio funcional, mas por poder ser identificada precocemente, possui potencial para prevenção e tratamento dos sintomas. Palavras-chave: Idoso fragilizado; Instituição de longa permanência para idosos; Saúde do idoso; Serviços de saúde para idosos.
\end{abstract}

\begin{abstract}
Objective: to determine the prevalence of frailty and associated factors in elderly people living in a long-term care facility. Method: cross-sectional descriptive study conducted with elderly residents in a long-term institution. The research was conducted through two questionnaires: a form for data collection regarding identification, sociodemographic and economic characteristics, and general health in the multidimensional assessment instrument for the elderly called Brazilian Functional Assessment Questionnaire (BOMFAQ) and Edmonton Frailty Scale, which assesses the level of frailty of elderly people. Results: 75 elderly were analyzed, among which 81,33\% were considered frail. It was found that the age group from 60 to 79 years old, and retirees had a significantly relevant relationship with frailty. Conclusion: most of the elderly evaluated presented frailty, which is a syndrome of multidimensional character, impacts on the personal, family and social life of the elderly, it can be a precursor of functional decline, but because it can be identified early, it has potential for prevention and treatment of symptoms.
\end{abstract}

Keywords: Frail elderly; Homes for the aged; Health of the elderly; Health services for the aged.

\section{Resumen}

Objetivo: determinar la prevalencia de fragilidad en personas mayores que viven en un centro de cuidados de larga duración. Método: estudio descriptivo transversal realizado con ancianos residentes en una institución de larga duración. Se utilizaron cuestionarios para evaluar las características sociodemográficas y económicas y la Edmonton Frail Scale. Resultados: De los 75 ancianos institucionalizados, el 81,33\% se consideró frágil. Se encontró que el grupo de edad de 60 a 79 años y jubilados tenían una relación significativamente relevante con la fragilidad. Conclusión: la mayoría de los ancianos evaluados presentó fragilidad, que es un síndrome de carácter multidimensional, impacta en la vida personal, familiar y social del anciano, puede ser una condición precursora del 
deterioro funcional, pero debido a que se puede identificar precozmente, tiene potencial para la prevención y el tratamiento de los síntomas.

Palabras clave: Anciano frágil; Institución de larga estancia para la tercera edad; Salud de los ancianos; Servicios de salud para personas mayores.

\section{Introdução}

O envelhecimento populacional e a longevidade são fenômenos mundiais, que levam ao aumento da prevalência das condições crônicas de saúde e podem expor as pessoas idosas a vivenciar a fragilidade e as alterações da funcionalidade global, aumentando, proporcionalmente, o grau de dependência física e social, interferindo na vida da pessoa idosa e no desempenho de suas atividades cotidianas de forma independente (Ministério da Saúde, 2006; Borges et al., 2013; Mello et al., 2014).

Entre os aspectos que repercutem na vida das pessoas idosas e que levam às dificuldades funcionais, destaca-se a fragilidade, a qual tem sido reconhecida, mundialmente, como indicador de saúde de pessoas idosas e como uma importante variável de avaliação da funcionalidade do idoso (Bergman et al., 2007; Gobbens et al., 2010; Morley et al., 2013). Trata-se de um fenômeno progressivo, com maior ocorrência a partir dos 80 anos, que traz mudanças significativas na vida do idoso (Mello et al., 2014).

Ainda não há consenso em relação à definição de fragilidade, sendo esse um conceito emergente, complexo e multifatorial utilizado para referenciar uma condição clínica desfavorável, que resulta de interação entre fatores clínicos e sociais, presentes na vida do idoso, gerando um impacto negativo que predispõe a uma deterioração progressiva e funcional aumentando a demanda de cuidados específicos (Veras et al, 2007; Andrade et al., 2012; Oliveira et al., 2012; Fabricio-Wehbe et al., 2013). Tais condições expõem os idosos e seus familiares a prejuízos práticos em suas vidas (Mello et al., 2014).

A fragilidade é entendida como a limitação que o idoso possui de manter a homeostase frente a estressores, que além de aumentar a vulnerabilidade com a idade, ocorre a redução de suas reservas fisiológicas, assim como, demandas sociais que poderão interferir na saúde do idoso e acarretar desfechos adversos, predispondo a dependência, às quedas recorrentes, à institucionalização, à hospitalização e à morte (Andrade et al., 2012; Oliveira et al., 2012; Fhon et al., 2012; Fabricio-Wehbe et al., 2013; Vieira et al., 2013). Essa condição está relacionada com três ou mais dos seguintes indicadores: perda de peso não intencional, baixo nível de atividade física, força de preensão palmar reduzida, velocidade da marcha reduzida e fadiga autorreferida (Linck et al., 2011).

Em geral, o nível de fragilidade interfere diretamente na capacidade do idoso para a manutenção das atividades básicas e instrumentais da vida diária, como também em suas relações sociais, fazendo com que se tornem dependentes de seus cuidadores e de seus familiares, gerando a necessidade de cuidados contínuos de longa duração. Esse aspecto tem aumentado a demanda por atendimento nas instituições de longa permanência (ILPI) (Veras et al, 2007; Oliveira et al., 2012).

A institucionalização por si, já é um fator para a ocorrência de fragilidade na população idosa (Bergman et al., 2007; Gobbens et al., 2010). As principais razões para que os idosos passem a residir em uma instituição de longa permanência estão relacionados ao aumento das demandas e necessidades de cuidado devido a comorbidades, aumento da dependência funcional e maior acometimento por agravos à saúde (Morley et al., 2013; Mello et al., 2014).

Mediante aos indicadores de fragilidade e os geradores de dependência, o setor de saúde nos últimos anos tem buscado meios para atender as necessidades dos idosos fragilizados, amparando esses idosos e seus familiares no processo de envelhecimento (Veras et al, 2007). A síndrome clínica referente à fragilidade no idoso surge como um fator importante na saúde pública devido às associações de desfechos negativos, e o quanto antes essa síndrome for identificada, ter-se-ão melhor prognóstico quando perceptível em seu início (Fabricio-Wehbe et al., 2013).

Nesse contexto, o idoso institucionalizado requer uma maior atenção frente às políticas de saúde, pois necessita de cuidados específicos em decorrência do avanço da idade, como também uma atuação para prevenção da fragilidade ou medidas 
que possam evitar o seu agravamento (Linck et al., 2011).

O presente estudo foi desenvolvido para determinar a prevalência de fragilidade em idosos residentes em uma instituição de longa permanência.

\section{Metodologia}

Estudo de corte transversal descritivo que foi desenvolvido em uma instituição de longa permanência para idosos (ILPI) em um município do Estado de Goiás. A ILPI investigada possui caráter não-governamental, sem fins lucrativos e está classificada como Modalidade III (ANVISA, 2005).

A população foi composta pelos 100 residentes na ILPI. Para inclusão no estudo foram considerados os seguintes critérios: ter mais de 60 anos de idade e ser residente na ILPI. Foram excluídos os idosos com tempo de institucionalização menor do que seis meses; e aqueles com diagnóstico médico de déficit cognitivo cujos familiares/tutores legais não residiam no município em estudo.

A coleta de dados ocorreu nas dependências da ILPI, entre $1^{\circ}$ de abril e 20 de maio de 2014, por meio de entrevista com os idosos e/ou cuidadores; observação, avaliação clínica e análise. A coleta constou da obtenção dos dados referentes à identificação, às características sociodemográficas e econômicas, à saúde geral, e ao nível de fragilidade por meio do prontuário médico e pela equipe de saúde da instituição.

Para a coleta de dados foram utilizados um formulário estruturado composto de duas seções: (1) dados de identificação, perfil sociodemográfico e econômico, e saúde geral, (2) a avaliação da fragilidade por meio da Escala de Fragilidade de Edmonton.

Para coleta dos dados de identificação, perfil social e saúde geral foi utilizado um questionário com questões objetivas relacionadas ao sexo, idade, estado conjugal, escolaridade, renda, aposentadoria, institucionalização e visitas, elaborado com base nas seções relacionadas à identificação pessoal, perfil social e condições de saúde do instrumento de avaliação multidimensional do idoso denominado "Brazilian Functional Assessment Questionnaire" (BOMFAQ) (Blay et al., 1988).

Para a análise do grau de fragilidade foi utilizada a Escala de Fragilidade de Edmonton (EFS), instrumento validado e traduzido no Brasil (Rolfson et al., 2006; Fabricio-Wehbe et al., 2009). Esse instrumento é composto por nove domínios: cognição, estado geral de saúde, independência funcional, suporte social, uso de medicamentos, nutrição, humor, continência e desempenho funcional. Cada domínio pode ser avaliado de 0 a 2 pontos cada, podendo alcançar o total de 18 pontos. A EFS detecta os seguintes níveis de fragilidade: 0 a 4 pontos, não apresenta fragilidade; 5 a 6 pontos, aparentemente vulnerável; 7 a 8 pontos, fragilidade leve; 9 a 10 pontos, fragilidade moderada e acima de 11 pontos, fragilidade severa.

Após a coleta de dados, todas as informações foram tabuladas em planilha do programa Excel para Mac ${ }^{\circledR} 2011$, versão 14.0.0, as variáveis foram codificadas e o banco foi validado para a análise da consistência interna, uma vez que foi digitado por três pessoas. Ao detectarem-se inconsistências, elas foram localizadas e as correções devidamente realizadas. Então a planilha foi exportada para o programa Software IBM Statistical Package for Social Science (SPSS) ${ }^{\circledR}$ for Windows, versão 20.0. Foram realizadas análises descritivas das variáveis contínuas mediante cálculo dos valores mínimos e máximos, médias, desvios padrão e medianas. As variáveis categóricas foram analisadas por meio de frequência absolutas e relativas.

Para uma melhor análise das variáveis dependentes (escores de fragilidade) e independentes (socioeconômicas, relacionadas à institucionalização e de saúde), os escores de fragilidade (Escala de Edmonton) foram agrupados em três grupos, ou seja, os idosos "não frágeis" e os "aparentemente frágil" foram classificados no grupo não-frágeis; os idosos com "fragilidade leve" e "fragilidade moderada" foram classificados como pré-frágeis e o restante dos idosos, ou seja, aqueles com "fragilidade severa" foram classificados como frágeis. Para as comparações entre as variáveis sociodemográficas, econômicas e condições gerais de saúde com os níveis de fragilidade utilizou-se o "qui-quadrado". 
A investigação obedeceu aos critérios estabelecidos pela Resolução 466/12 do Conselho Nacional de Saúde (Ministério da Saúde, 2012) e foi aprovada pelo Comitê de Ética da Pontifícia Universidade Católica de Goiás com o CAAE 269460143.3.0000.0037 e parecer de número 537.642.

\section{Resultados}

Do total de 100 idosos residentes na ILPI, 75 foram incluídos. A média de idade dos participantes foi de $76,3 \pm 8,0$ anos, sendo 72,0\% deles do sexo masculino, 76,0\% não tinham companheiros, 64,0\% não possuíam estudo e 90,7\% eram aposentados. Os níveis de fragilidade identificados por meio da Escala de Fragilidade de Edmonton estão descritos no Gráfico 1. Observou-se que a prevalência de fragilidade entre os 75 participantes foi de $81,33 \%$.

Gráfico 1. Distribuição dos idosos segundo os níveis de fragilidade de acordo com a Escala de Fragilidade de Edmonton (n=75). Rio Verde, GO, Brasil, 2014.

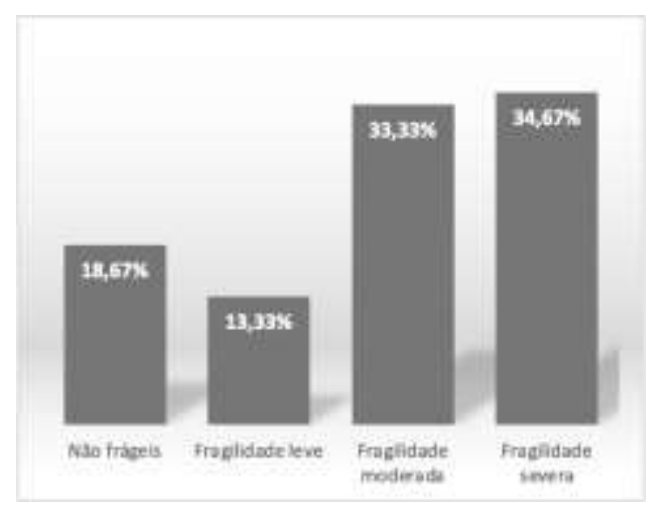

Fonte: Autores.

A Tabela 1 apresenta a distribuição da classificação da EFE segundo as variáveis sóciodemográficas dos idosos avaliados, dentre as quais somente faixa etária $(\mathrm{p}=0,016)$ e tipo de renda $(\mathrm{p}=0,028)$ apresentaram associação significativa com os níveis de fragilidade. 
Tabela 1. Distribuição dos níveis de fragilidade de acordo com a Escala de Fragilidade de Edmonton segundo as variáveis sociodemográficas (n=75). Rio Verde, GO, Brasil, 2014.

\begin{tabular}{|c|c|c|c|c|c|}
\hline Variáveis & $\begin{array}{c}\text { Não- Frágil } \\
\mathrm{n}(\%)\end{array}$ & $\begin{array}{l}\text { Pré- Frágil } \\
\mathrm{n}(\%)\end{array}$ & $\begin{array}{l}\text { Frágil } \\
\mathrm{n}(\%)\end{array}$ & $\begin{array}{l}\text { Total } \\
\mathrm{n}(\%)\end{array}$ & $p^{*}$ \\
\hline \multicolumn{6}{|l|}{ Idade (em anos) } \\
\hline 60 I---I 79 & $1(1,9)$ & $12(23,1)$ & $39(75,0)$ & $52(69,3)$ & \multirow{2}{*}{0,016} \\
\hline$\geq 80$ & $4(17,4)$ & $8(34,8)$ & $11(47,8)$ & $23(30,7)$ & \\
\hline \multicolumn{6}{|l|}{ Sexo } \\
\hline Feminino & $3(14,3)$ & $7(33,3)$ & $11(52,4)$ & $21(28,0)$ & \multirow[t]{2}{*}{0,141} \\
\hline Masculino & $2(3,7)$ & $13(24,1)$ & $39(72,2)$ & $54(72,0)$ & \\
\hline \multicolumn{6}{|l|}{ Estado Conjugal } \\
\hline Sem companheiro & $5(8,8)$ & $16(28,1)$ & $36(48,0)$ & $57(76,0)$ & \multirow[t]{2}{*}{0,334} \\
\hline Com companheiro & $0(0,0)$ & $4(22,2)$ & $14(77,8)$ & $18(24,0)$ & \\
\hline \multicolumn{6}{|l|}{ Escolaridade } \\
\hline Com instrução & $2(7,4)$ & $9(33,3)$ & $16(59,3)$ & $27(36,0)$ & \multirow[t]{2}{*}{0,581} \\
\hline Sem instrução & $3(6,3)$ & $11(22,9)$ & $34(70,8)$ & $48(64,0)$ & \\
\hline \multicolumn{6}{|l|}{ Renda } \\
\hline Sim & $5(6,8)$ & $19(26,0)$ & $49(67,1)$ & $73(97,3)$ & \multirow[t]{2}{*}{0,725} \\
\hline Não & $0(0,0)$ & $1(50,0)$ & $1(50,0)$ & $2(2,7)$ & \\
\hline \multicolumn{6}{|l|}{ Tipo de renda } \\
\hline Aposentadoria & $3(4,4)$ & $19(27,9)$ & $46(67,6)$ & $68(90,7)$ & \multirow[t]{3}{*}{0,028} \\
\hline Pensão & $2(40,0)$ & $0(0,0)$ & $3(60,0)$ & $5(6,7)$ & \\
\hline $\mathrm{BPC}^{* *}$ & $0(0,0)$ & $1(50,0)$ & $1(50,0)$ & $2(2,7)$ & \\
\hline \multicolumn{6}{|l|}{$\begin{array}{l}\text { Motivo da } \\
\text { aposentadoria }\end{array}$} \\
\hline Tempo de serviço & $0(0,0)$ & $0(0,0)$ & $2(2,7)$ & $2(2,7)$ & \multirow[t]{3}{*}{0,308} \\
\hline Idade & $5(8,8)$ & $13(22,8)$ & $39(68,4)$ & $57(76,0)$ & \\
\hline Questão de saúde & $0(0,0)$ & $7(43,8)$ & $9(56,3)$ & $16(21,1)$ & \\
\hline
\end{tabular}

*Teste do Qui-quadrado; **Benefício de Proteção Continuada. Fonte: Autoras.

A Tabela 2 apresenta a distribuição da classificação da EFE segundo as variáveis relacionadas à institucionalização dos idosos, dentre as quais não foi encontrada nenhuma associação entre as referidas variáveis e a fragilidade nos idosos avaliados.

Tabela 2. Distribuição dos níveis de fragilidade de acordo com a Escala de Fragilidade de Edmonton segundo as variáveis relacionadas à institucionalização $(\mathrm{n}=75)$. Rio Verde, GO, Brasil, 2014.

\begin{tabular}{|c|c|c|c|c|c|}
\hline \multirow[t]{2}{*}{ Variáveis } & $\begin{array}{l}\text { Não - } \\
\text { Frágil } \\
\end{array}$ & Pré- Frágil & Frágil & \multirow[t]{2}{*}{ Total } & \multirow[t]{2}{*}{$p^{* *}$} \\
\hline & $\mathrm{n}(\%)$ & $\mathrm{n}(\%)$ & $\mathrm{n}(\%)$ & & \\
\hline \multicolumn{6}{|c|}{$\begin{array}{l}\text { Tempo de institucionalização } \\
\text { (em anos) }\end{array}$} \\
\hline$<10$ anos & $3(5,3)$ & $13(22,8)$ & $41(71,9)$ & $57(76,0)$ & 0,221 \\
\hline$>10$ anos & $2(11,1)$ & $7(38,9)$ & $9(50,0)$ & $18(24,0)$ & \\
\hline \multicolumn{6}{|c|}{$\begin{array}{l}\text { Com quem morava antes da } \\
\text { institucionalização }\end{array}$} \\
\hline Sozinho & $1(5,0)$ & $4(20,0)$ & $15(75,0)$ & $20(26,7)$ & 0,653 \\
\hline Com outras pessoas & $4(7,3)$ & $16(29,1)$ & $35(63,6)$ & $55(73,3)$ & \\
\hline \multicolumn{6}{|l|}{ Visitas } \\
\hline Recebe & $4(7,8)$ & $15(29,4)$ & $32(62,7)$ & $51(68,0)$ & 0,563 \\
\hline Não recebe & $1(4,2)$ & $5(20,8)$ & $18(75,0)$ & $24(32,0)$ & \\
\hline \multicolumn{6}{|l|}{ Quem visita } \\
\hline Filhos & $2(7,4)$ & $5(18,5)$ & $20(74,1)$ & $27(36,0)$ & 0,489 \\
\hline Outros & $3(6,3)$ & $15(31,3)$ & $30(62,5)$ & $48(64,0)$ & \\
\hline
\end{tabular}

*Escala de Fragilidade de Edmonton; **Teste do Qui-quadrado. Fonte: Autoras.

A Tabela 3 apresenta a distribuição da classificação da EFE segundo as variáveis relacionadas às características gerais de saúde dos idosos, as quais também não obtiveram associação com os níveis de fragilidade. 
Tabela 3. Distribuição dos níveis de fragilidade de acordo com a Escala de Fragilidade de Edmonton segundo as características gerais de saúde (n=75). Rio Verde, GO, Brasil, 2014.

\begin{tabular}{lccccc}
\hline \multicolumn{1}{c}{ Variáveis } & Não- Frágil & Pré- Frágil & Frágil & Total (n\%) & $\mathrm{p}^{*}$ \\
\hline $\begin{array}{l}\text { Percepção da Saúde } \\
\quad \text { Satisfatória }\end{array}$ & $5(7,7)$ & $18(27,7)$ & $42(64,6)$ & $65(86,7)$ & 0,530 \\
$\quad$ Insatisfatória & $0(0,0)$ & $2(20,0)$ & $8(80,0)$ & $10(13,3)$ & \\
$\begin{array}{l}\text { Saúde comparada com } \\
\text { outros indivíduos }\end{array}$ & & & & & \\
$\quad$ Melhor & $2(10,0)$ & $5(25,0)$ & $13(65,0)$ & $20(26,7)$ & 0,659 \\
$\quad$ Igual & $3(7,0)$ & $13(30,2)$ & $27(62,8)$ & $43(57,3)$ & \\
$\quad$ Pior & $0(0,0)$ & $2(16,7)$ & $10(83,3)$ & $12(16,0)$ & \\
$\quad \begin{array}{l}\text { Número de medicamentos } \\
\quad<5\end{array}$ & $3(8,8)$ & $8(23,5)$ & $23(67,6)$ & $34(45,3)$ & 0,714 \\
$\quad 25$ & $2(4,9)$ & $12(29,3)$ & $27(65,9)$ & $41(54,7)$ & \\
$\quad \begin{array}{l}\text { Comorbirdades relatadas } \\
\quad<3\end{array}$ & $0(0,0)$ & $4(33,3)$ & $8(6,7)$ & $12(16,0)$ & 0,551 \\
$\quad \geq 3$ & $5(7,9)$ & $16(25,4)$ & $42(66,7)$ & $63(84,0)$ & \\
\hline
\end{tabular}

*Teste do Qui-quadrado. Fonte: Autores.

\section{Discussão}

Verificou-se a elevada prevalência de fragilidade em idosos institucionalizados avaliados, o que condiz com o perfil desta população no contexto brasileiro (Borges et al., 2013; Fhon et al., 2012). A fragilidade corresponde a um desfecho esperado frente ao processo da institucionalização das pessoas idosas (Lana e Schneider, 2014; Remor et al., 2014). A elevada taxa de fragilidade em idosos institucionalizados demonstra a necessidade de que as instituições de longa permanência para idosos adotem modelos de cuidado que promovam intervenções para melhorar a funcionalidade global do idoso, promovendo ações que possibilitem o envelhecimento ativo e saudável, melhorando a capacidade física e mental da pessoa idosa, além de prevenir doenças e o surgimento ou agravamento da fragilidade(Araújo \& Ceolin, 2007; Linck et al., 2011; Silva et al., 2011; Storti et al., 2014).

A maior a prevalência de fragilidade no grupo etário correspondente a 60 a 79 anos de idade, a qual se assemelha àquelas identificadas em outros estudos (Araújo \& Ceolin, 2007; Silva et al., 2011; Salmazo-Silva et al., 2012). No entanto, a maioria dos estudos revela que os níveis de fragilidade são mais elevados naqueles idosos que encontram-se acima dos 80 anos de idade, uma vez que as faixas etárias mais longevas relacionam-se à redução da capacidade funcional, surgimento de doenças crônicas não-transmissíveis devido às consequências do envelhecimento (Mazo et al., 2007; Borges et al., 2015; BandeenRoche et al., 2015).

A população masculina apresentou maior prevalência de fragilidade, em discrepância com a maioria das pesquisas com temática semelhante. Segundo estes estudos, devido a maior expectativa de vida, menores taxas de mortalidade e maior utilização e procura por serviços de saúde, fazem com que as mulheres alcancem maiores faixas etárias e sejam mais afetadas pela síndrome da fragilidade (Rosa et al., 2003; Creutzberg et al., 2007; Hilmer et al., 2009).

Nota-se que a maioria dos participantes não tinha companheiros $(76,0 \%)$, e o predomínio de idosos frágeis associados a essa condição (48,0\%). De acordo com estudos prévios, a institucionalização de idosos e ausência de companheiros, correspondem a um fator de risco para a fragilidade e também para a própria institucionalização (Mello et al., 2014; Vasconcelos et al., 2020).

Entre os idosos pesquisados, $64 \%$ não possuíam estudo, e 70,8\% desses idosos foram classificados como frágeis. 
Pesquisadores descrevem que idosos com baixo nível de escolaridade estão predispostos a apresentarem problemas de saúde, pelo comprometimento do autocuidado (Rosa et al., 2003; Creutzberg et al., 2007; Hilmer et al., 2009). Nesse sentido, as intervenções educativas são fundamentais de modo a orientar e acompanhar o desenvolvimento do idoso para que ele compreenda sua situação de saúde e aceite a terapêutica. Empoderar e acolher o idoso nesse caso é fundamental, como também estimular a autonomia e independência durante as atividades cotidianas, permitindo durante a institucionalização, uma independência física e comportamental (Bergman et al., 2007; Hilmer et al, 2009; Remor et al., 2011).

Entre os participantes, a maior parte eram aposentados, essa variável associa-se ao idoso frágil, em especial aqueles que foram aposentados por idade $(68,4 \%)$. Há o predomínio de idosos institucionalizados aposentados, sendo a aposentadoria fonte de renda de grande parte dos idosos (Fochat et al., 2012; Fluetti et al., 2018).

Percebeu-se que existe maior prevalência de fragilidade entre os idosos que residiam na ILPI há mais tempo, corroborando com estudo realizado em Ubá-MG, em que os autores afirmam que quanto maior o tempo de institucionalização, maior a fragilidade nos idosos (Ferrareti et al., 2020).

Muitos idosos buscam a institucionalização como alternativa por fatores como não ter condições físicas e psicossociais para viverem sozinhos, como também não terem suporte familiar. Observa-se que com o envelhecimento, acarreta o aparecimento da síndrome da fragilidade, dessa forma os idosos buscam refúgio em ILPI para melhor qualidade de vida (Lisboa et al., 2012).

Observou-se que antes de morarem na ILPI, os idosos referiram que moravam com outras pessoas. As mudanças nos arranjos familiares que ocorrem com o envelhecimento, podem levar os idosos a necessitar de cuidados e de atenção especializada, e assim os familiares optam pela institucionalização, como meio de proporcionar uma qualidade de vida ao idoso (Borges et al., 2015).

Quanto às visitas, a maioria dos idosos recebiam visitas regulares na ILPI $(62,7 \%)$, sendo a maior parte das visitas feitas pelos filhos. Dessa forma, percebe-se que o arranjo familiar se mantém mesmo com institucionalização (Fochat et al., 2012; Fluetti et al., 2018).

A percepção de saúde entre os idosos frágeis caracterizaram sua saúde geral como insatisfatória (80,0\%). Observa-se que grande parte dos idosos nesse estudo, consideram sua saúde pior quando comparada a outros indivíduos, devido ao número elevado de medicações e quantidade de morbidades que possuem. Estudos apontam que maior número de comorbidades e o uso de várias medicações, acarretam piores condições de saúde, alteram a manutenção do autocuidado e aumentam o estado de vulnerabilidade do idoso (Fluetti et al., 2018; Santiago \& Luz, 2016; Oliveira et al., 2021).

Os resultados apontaram que $84 \%$ dos idosos possuíam mais de três comorbidades e 54,7\% tomavam mais de cinco medicações. Apesar de não ter sido observada diferença em termos do nível de fragilidade é importante considerar as evidências de que idosos institucionalizados possuem um perfil diferenciado de idosos ambulatoriais, e somada à fragilidade a necessidade de medicações aumenta em relação à vida de idosos frágeis (Borges et al., 2013; Remor et al., 2011; Araujo \& Ceolim, 2007; Luchetti et al., 2010).

A avaliação do nível de fragilidade em idosos tem sido considerado um importante indicador do processo de envelhecimento é variável fundamental para o planejamento das intervenções em saúde, em especial em populações idosas vulneráveis tais quais as que se encontram institucionalizadas. Para este processo, é importante a utilização de instrumentos validados para a realidade do local e da população a ser estudada. Neste estudo, para avaliar o nível de fragilidade dos idosos foi utilizada a Escala de Fragilidade de Edmonton assim como em outros estudos (Giacomini et al., 2020; Guedes et al., 2020; Santos et al., 2020). Existem outros instrumentos para o mesmo fim utilizados em estudos com o objetivo de mensurar os níveis de fragilidade entre idosos, tais como o Indicador de Fragilidade de Tilburg (Carneiro et al., 2017; Jesus et al., 2017), Indicador de Fragilidade de Groningen (Borges et al., 2013), Fenótipo de Fragilidade de Fried (Fried et al., 2001), Índice de 
Vulnerabilidade Clínico-funcional (ICVF-20) (Moraes et al., 2016) e Instrumento Autorreferido de Fragilidade (Nunes et al., 2015), entre outros.

Os resultados devem ser interpretados mediante suas limitações em relação ao tipo de estudo, à amostra e aos instrumentos de coleta utilizados. Uma vez que se trata de uma investigação do tipo transversal, não é possível o estabelecimento das relações causais entre as variáveis apresentadas. Para que estes efeitos sejam avaliados se faz necessária a realização de estudos subsequentes de caráter longitudinal.

Em relação à amostra do estudo, a qual foi composta de idosos residentes em uma única instituição de longa permanência, pode ter ocorrido uma homogeneização da amostra do estudo quanto à quantidade e qualidade de cuidado e assistência à saúde recebida. Este fato pode ter ocasionado um viés tal que o grupo amostral não corresponda à realidade da população idosa institucionalizada que apresenta fragilidade.

A qualidade das informações coletadas por meio dos questionários utilizados também pode gerar limitações a esse estudo. Apesar dos mesmos se constituírem de instrumentos validados e adaptados para o contexto de idosos institucionalizados, e ainda terem sido aplicados por profissionais de saúde treinados em sua aplicação, podem gerar informações que tenham sido influenciadas pela opinião pessoal do aplicador.

\section{Conclusão}

Identificou-se a elevada prevalência de fragilidade em idosos institucionalizados, principalmente na faixa etária de 60 a 79 anos de idade e naqueles que se encontram fora de suas atividades laborais. A avaliação da fragilidade é fundamental para o estabelecimento precoce de intervenções que favoreçam a autonomia, independência e a participação social do idoso, promovendo não apenas um atendimento de reabilitação, mas também de prevenção à síndrome por meio da promoção e educação em saúde.

Os resultados apontam a necessidade da realização de estudos nacionais e multicêntricos que tenham como objetivo verificar como tem sido a implementação dos cuidados nesses importantes espaços de cuidados de modo a gerar indicadores do processo e da qualidade do cuidado nessas instituições, bem como as soluções em saúde implementadas para reabilitação de idosos frágeis.

Sugere-se ainda a realização de estudos, preferencialmente de caráter longitudinal, que possuam uma amostra maior de idosos institucionalizados, provindos de instituições distintas, os quais possam avaliar a existência de correlações entre a síndrome da fragilidade, faixa etária e afastamento das atividades laborais, e a possibilidade de se intervir e reverter o processo de fragilização.

\section{Referências}

Andrade, A. do N., Fernandes, M. das G. M., Nóbrega, M. M. L. da, Garcia, T. R., \& Costa, K. N. de F. M. (2012). Análise do conceito fragilidade em idosos. Texto \& Contexto - Enfermagem, 21, 748-756. https://doi.org/10.1590/S0104-07072012000400004.

ANVISA. Agência Nacional de Vigilância Sanitária. (2005). Resolução da Diretoria Colegiada- RDC/ANVISA nº 283 , de 26 de setembro de 2005. Aprova o Regulamento Técnico que define normas de funcionamento para as Instituições de Longa Permanência para Idosos, de caráter residencial.

Araújo, M. O. P. H. de, \& Ceolim, M. F. (2007). Avaliação do grau de independência de idosos residentes em instituições de longa permanência. Revista da Escola de Enfermagem da USP, 41(3), 378-385. https://doi.org/10.1590/S0080-62342007000300006

Bandeen-Roche, K., Seplaki, C. L., Huang, J., Buta, B., Kalyani, R. R., Varadhan, R., Xue, Q.-L., Walston, J. D., \& Kasper, J. D. (2015). Frailty in older adults: A nationally representative profile in the United States. The Journals of Gerontology. Series A, Biological Sciences and Medical Sciences, 70(11), 1427-1434. https://doi.org/10.1093/gerona/glv133

Bergman, H., Ferrucci, L., Guralnik, J., Hogan, D. B., Hummel, S., Karunananthan, S., \& Wolfson, C. (2007). Frailty: An emerging research and clinical paradigm--issues and controversies. The Journals of Gerontology. Series A, Biological Sciences and Medical Sciences, 62(7), 731-737. https://doi.org/10.1093/gerona/62.7.731 
Blay, S. L., Ramos, L. R., \& Mari, J. de J. (1988). Validity of a Brazilian version of the Older Americans Resources and Services (Oars) mental health screening questionnaire. Journal of the American Geriatrics Society, 36(8), 687-692. https://doi.org/10.1111/j.1532-5415.1988.tb07169.x

Borges, C. L., Fernandes, B. K. C., Clares, J. W. B., Cavalcante, M. L. S. N., Leite, S. F. P., Junior, A. A. P., \& Freitas, M. C. de. (2019). Tradução e adaptação transcultural do Groningen Frailty Indicator para idosos brasileiros. Journal of Health \& Biological Sciences, 7(3(Jul-Set)), $242-247$. https://doi.org/10.12662/2317-3076jhbs.v7i3.2230.p242-247.2019

Borges, C. L., Silva, M. J. da, Clares, J. W. B., Bessa, M. E. P., \& Freitas, M. C. de. (2013). Avaliação da fragilidade de idosos institucionalizados. Acta Paulista de Enfermagem, 26(4), 318-322. https://doi.org/10.1590/S0103-21002013000400004

Borges, C. L., Silva, M. J. da, Clares, J. W. B., Nogueira, J. D. M., \& Freitas, M. C. de. (2015). Características sociodemográficas e clínicas de idosos institucionalizados: Contribuições para o cuidado de enfermagem. Revista Enfermagem UERJ, 23(3). https://doi.org/10.12957/reuerj.2015.4214

Carneiro, J. A., Cardoso, R. R., Durães, M. S., Guedes, M. C. A., Santos, F. L., Costa, F. M. da, \& Caldeira, A. P. (2017). Frailty in the elderly: Prevalence and associated factors. Revista Brasileira de Enfermagem, 70(4), 747-752. https://doi.org/10.1590/0034-7167-2016-0633

Creutzberg, M., Gonçalves, L. H. T., Sobottka, E. A., \& Ojeda, B. S. (2007). A Instituição de Longa Permanência para Idosos e o sistema de saúde. Revista Latino-Americana de Enfermagem, 15(6), 1144-1149. https://doi.org/10.1590/S0104-11692007000600014

Fabrício-Wehbe, S. C. C., Cruz, I. R., Haas, V. J., Diniz, M. A., Dantas, R. A. S., \& Rodrigues, R. A. P. (2013). Reproducibility of the Brazilian version of the Edmonton Frail Scale for elderly living in the community. Revista Latino-Americana de Enfermagem, 21(6), 1330-1336. https://doi.org/10.1590/01041169.2933 .2371

Fabrício-Wehbe, S. C. C., Schiaveto, F. V., Vendrusculo, T. R. P., Haas, V. J., Dantas, R. A. S., \& Rodrigues, R. A. P. (2009). Cross-cultural adaptation and validity of the «Edmonton Frail Scale-EFS» in a Brazilian elderly sample. Revista Latino-Americana de Enfermagem, 17(6), 1043-1049. https://doi.org/10.1590/S0104-11692009000600018

Ferrarez, M. L., Martins, M. C. de L., Lima, G. E. G., Trevizani, G. A., \& Martinho, K. O. (2020). Análise da Síndrome da fragilidade em idosos institucionalizados / Analysis of frailty syndrome in institutionalized elderly. Brazilian Journal of Health Review, 3(6), 19633-19646. https://doi.org/10.34119/bjhrv3n6-339

Fhon, J. R. S., Diniz, M. A., Leonardo, K. C., Kusumota, L., Haas, V. J., \& Rodrigues, R. A. P. (2012). Síndrome de fragilidade relacionada à incapacidade funcional no idoso. Acta Paulista de Enfermagem, 25, 589-594. https://doi.org/10.1590/S0103-21002012005000016

Fluetti, M. T., Fhon, J. R. S., Oliveira, A. P. de, Chiquito, L. M. O., \& Marques, S. (2018). The frailty syndrome in institutionalized elderly persons. Revista Brasileira de Geriatria e Gerontologia, 21(1), 60-69. https://doi.org/10.1590/1981-22562018021.170098

Fochat, R. C., Horsth, R. B. de O., Vianna, C. L. C., Raposo, N. R. B., Vieira, R. de C. P. A., \& Chicourel, E. L. (2012). Perfil sociodemográfico de idosos frágeis institucionalizados em juiz de fora - minas gerais. Revista de APS, 15(2). https://periodicos.ufjf.br/index.php/aps/article/view/14838

Fried, L. P., Tangen, C. M., Walston, J., Newman, A. B., Hirsch, C., Gottdiener, J., Seeman, T., Tracy, R., Kop, W. J., Burke, G., McBurnie, M. A., \& Cardiovascular Health Study Collaborative Research Group. (2001). Frailty in older adults: Evidence for a phenotype. The Journals of Gerontology. Series A, Biological Sciences and Medical Sciences, 56(3), M146-156. https://doi.org/10.1093/gerona/56.3.m146

Giacomini, S. B. L., Fhon, J. R., \& Rodrigues, R. A. P. (2020). Fragilidade e risco de queda em idosos que vivem no domicílio. Acta Paulista de Enfermagem, 33, eAPE20190124. https://doi.org/10.37689/acta-ape/2020AO0124

Gobbens, R. J. J., van Assen, M. A. L. M., Luijkx, K. G., Wijnen-Sponselee, M. T., \& Schols, J. M. G. A. (2010). Determinants of frailty. Journal of the American Medical Directors Association, 11(5), 356-364. https://doi.org/10.1016/j.jamda.2009.11.008

Guedes, R. de C., Dias, R., Neri, A. L., Ferriolli, E., Lourenço, R. A., \& Lustosa, L. P. (2020). Frailty syndrome in Brazilian older people: A population based study. Ciência \& Saúde Coletiva, 25(5), 1947-1954. https://doi.org/10.1590/1413-81232020255.21582018

Hilmer, S. N., Perera, V., Mitchell, S., Murnion, B. P., Dent, J., Bajorek, B., Matthews, S., \& Rolfson, D. B. (2009). The assessment of frailty in older people in acute care. Australasian Journal on Ageing, 28(4), 182-188. https://doi.org/10.1111/j.1741-6612.2009.00367.x

Jesus, I. T. M. de, Orlandi, A. A. dos S., Grazziano, E. da S., \& Zazzetta, M. S. (2017). Fragilidade de idosos em vulnerabilidade social. Acta Paulista de Enfermagem, 30(6), 614-620. https://doi.org/10.1590/1982-0194201700088

Lana, L. D., \& Schneider, R. H. (2014). Síndrome de fragilidade no idoso: Uma revisão narrativa. Revista Brasileira de Geriatria e Gerontologia, 17(3), 673680. https://doi.org/10.1590/1809-9823.2014.12162

Linck, C. de L., \& Crossetti, M. da G. O. (2011). Fragilidade no idoso: O que vem sendo produzido pela enfermagem. Revista Gaúcha de Enfermagem, 32(2), 385-393. https://doi.org/10.1590/S1983-14472011000200024

Lisboa, C. R., \& Chianca, T. C. M. (2012). Perfil epidemiológico, clínico e de independência funcional de uma população idosa institucionalizada. Revista Brasileira de Enfermagem, 65(3), 482-488. https://doi.org/10.1590/S0034-71672012000300013

Lucchetti, G., Granero, A. L., Pires, S. L., \& Gorzoni, M. L. (2010). Fatores associados à polifarmácia em idosos institucionalizados. Revista Brasileira de Geriatria e Gerontologia, 13(1), 51-58. https://doi.org/10.1590/S1809-98232010000100006

Mazo, G., Liposcki, D., Ananda, C., \& Prevê, D. (2007). Condições de saúde, incidência de quedas e nível de atividade física dos idosos. Revista Brasileira de Fisioterapia, 11(6). https://doi.org/10.1590/S1413-35552007000600004

Mello, A. de C., Engstrom, E. M., \& Alves, L. C. (2014). Health-related and socio-demographic factors associated with frailty in the elderly: A systematic literature review. Cadernos de Saúde Pública, 30(6), 1143-1168. https://doi.org/10.1590/0102-311X00148213 
Ministério da Saúde.(2006). Envelhecimento e saúde da pessoa idosa. Brasília: Ministério da Saúde, (Cadernos de Atenção Básica, n. 19) (Série A. Normas e Manuais Técnicos).

Ministério da Saúde. (2012). Resolução CNS nº 466 de 12 de dezembro de 2012. Aprova as diretrizes e normas regulamentadoras das pesquisas envolvendo seres humanos.

Moraes, E. N. de, Carmo, J. A. do, Moraes, F. L. de, Azevedo, R. S., Machado, C. J., \& Montilla, D. E. R. (2016). Clinical-Functional Vulnerability Index-20 (IVCF-20): Rapid recognition of frail older adults. Revista de Saúde Pública, 50(0). https://doi.org/10.1590/s1518-8787.2016050006963

Morley, J. E., Vellas, B., van Kan, G. A., Anker, S. D., Bauer, J. M., Bernabei, R., Cesari, M., Chumlea, W. C., Doehner, W., Evans, J., Fried, L. P., Guralnik, J. M., Katz, P. R., Malmstrom, T. K., McCarter, R. J., Gutierrez Robledo, L. M., Rockwood, K., von Haehling, S., Vandewoude, M. F., \& Walston, J. (2013). Frailty consensus: A call to action. Journal of the American Medical Directors Association, 14(6), 392-397. https://doi.org/10.1016/j.jamda.2013.03.022

Nunes, D. P., Duarte, Y. A. de O., Santos, J. L. F., \& Lebrão, M. L. (2015). Screening for frailty in older adults using a self-reported instrument. Revista de Saúde Pública, 49(0). https://doi.org/10.1590/S0034-8910.2015049005516

Oliveira, D. R., Bettinelli, L. A., Pasqualotti, A., Corso, D., Brock, F., \& Erdmann, A. L. (2013). Prevalence of frailty syndrome in old people in a hospital institution. Revista Latino-Americana de Enfermagem, 21, 891-898. https://doi.org/10.1590/S0104-11692013000400009

Oliveira, P. H. de, \& Mattos, I. E. (2012). Prevalência e fatores associados à incapacidade funcional em idosos institucionalizados no Município de Cuiabá, Estado de Mato Grosso, Brasil, 2009-2010. Epidemiologia e Serviços de Saúde, 21(3), 395-406. https://doi.org/10.5123/S1679-49742012000300005

Oliveira, P. R. C., Rodrigues, V. E. S., Oliveira, A. K. L. de, Oliveira, F. G. L., Rocha, G. A., \& Machado, A. L. G. (2021). Fatores associados à fragilidade em idosos acompanhados na Atenção Primária à Saúde. Escola Anna Nery, 25. https://doi.org/10.1590/2177-9465-EAN-2020-0355

Remor, C. B., Bós, A. J. G., \& Werlang, M. C. (2011). Características relacionadas ao perfil de fragilidade no idoso. 21 (3), 107-112.

Rolfson, D. B., Majumdar, S. R., Tsuyuki, R. T., Tahir, A., \& Rockwood, K. (2006). Validity and reliability of the edmonton frail scale. Age and Ageing, 35(5), 526-529. https://doi.org/10.1093/ageing/afl041

Rosa, T. E. da C., Benício, M. H. D., Latorre, M. do R. D. de O., \& Ramos, L. R. (2003). Fatores determinantes da capacidade funcional entre idosos. Revista de Saúde Pública, 37(1), 40-48. https://doi.org/10.1590/S0034-89102003000100008

Salmazo-Silva, H., Lima-Silva, T. B., Barros, T. C. de, Oliveira, E. M. de, Ordonez, T. N., Carvalho, G., \& Almeida, E. B. de. (2012). Vulnerabilidade na velhice: Definição e intervenções no campo da Gerontologia. Revista Kairós-Gerontologia, 15, 97-116. https://doi.org/10.23925/2176901X.2012v15iEspecial13p97-116

Santiago, L. M., Luz, L. L., Silva, J. F. S. da, Oliveira, P. H. de, Carmo, C. N. do, \& Mattos, I. E. (2016). Socio-demographic and health conditions of institutionalized elders in cities of the Southeast and Middle West regions of Brazil. Geriatrics, Gerontology and Aging, 10(2), 86-92. http://ggaging.com/details/369/pt-BR/socio-demographic-and-health-conditions-of-institutionalized-elders-in-cities-of-the-southeast-and-middle-west-regionsof-brazil

Santiago, L. M., \& Mattos, I. E. (2014). Prevalência e fatores associados à fragilidade em idosos institucionalizados das regiões Sudeste e Centro-Oeste do Brasil. Revista Brasileira de Geriatria e Gerontologia, 17(2), 327-337. https://doi.org/10.1590/S1809-98232014000200010

Santos, R. C. dos, Menezes, R. M. P. de, Araújo, G. K. N. de, Marcolino, E. de C., Xavier, A. G., Gonçalves, R. G., \& Souto, R. Q. (2020). Síndrome da fragilidade e fatores associados em idosos no pronto atendimento. Acta Paulista de Enfermagem, 33, eAPE20190159. https://doi.org/10.37689/actaape/2020AO0159

Silva, V. A. da, Souza, K. L. de, \& D’Elboux, M. J. (2011). Incontinência urinária e os critérios de fragilidade em idosos em atendimento ambulatorial. Revista da Escola de Enfermagem da USP, 45(3), 672-678. https://doi.org/10.1590/S0080-62342011000300018

Storti, L. B., Fabrício-Whebe, S. C. C., Kusumota, L., Rodrigues, R. A. P., \& Marques, S. (2013). Fragilidade de idosos internados na clínica médica da unidade de emergência de um hospital geral terciário. Texto \& Contexto - Enfermagem, 22(2), 452-459. https://doi.org/10.1590/S0104-07072013000200022

Vasconcelos, A. C. de S. e, Marques, A. P. de O., Leite, V. M. M., Carvalho, J. C., \& Costa, M. L. G. da. (2020). Prevalência de fragilidade e fatores associados em idosos pós-acidente vascular cerebral. Revista Brasileira de Geriatria e Gerontologia, 23(5), e200322. https://doi.org/10.1590/198122562020023.200322

Veras, R. P., Caldas, C. P., Coelho, F. D., \& Sanchez, M. A. (2007). Promovendo a Saúde e Prevenindo a Dependência: Identificando indicadores de fragilidade em idosos independentes. Revista Brasileira de Geriatria e Gerontologia, 10(3), 355-370. https://doi.org/10.1590/1809-9823.2007.10038

Vieira, R. A., Guerra, R. O., Giacomin, K. C., Vasconcelos, K. S. de S., Andrade, A. C. de S., Pereira, L. S. M., Dias, J. M. D., \& Dias, R. C. (2013). Prevalência de fragilidade e fatores associados em idosos comunitários de Belo Horizonte, Minas Gerais, Brasil: Dados do estudo FIBRA. Cadernos de Saúde Pública, 29(8), 1631-1643. https://doi.org/10.1590/S0102-311X2013001200015 